\section{Fab direction for AAV}

AAV vectors are promising candidates for human gene therapy. The inability of AAV to bind cells lacking the heparan sulfate proteoglycan, however, has limited the range of cells that can be targeted. Using a bispecific antibody that recognizes the platelet-specific $\alpha_{\mathrm{IIb}} \beta_{3}$ integrin and the AAV capsid structure, Bartlett et al. have successfully transduced megakaryocyte cell lines recalcitrant to infection by wild-type AAV (p. 181). Interestingly, the bispecific antibody also appeared to reduce the endogenous tropism of the AAV vector for heparan sulfate bearing cells.

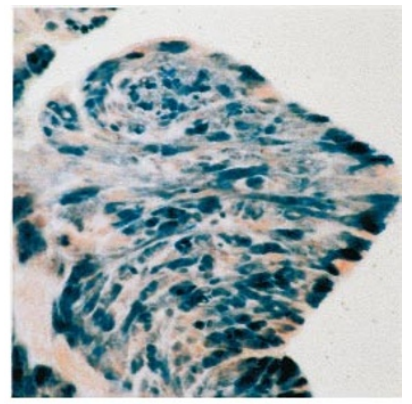

On p. 165, Redfern et al. report the creation of transgenic mice that inducibly express a $\mathrm{G}_{\mathrm{i}}$ coupled $\kappa$ opioid receptor altered so as to be nonreponsive to its natural ligand, but responsive to the small-molecule spiradoline. Using the tetracycline transactivator system, they induced expression of this engineered receptor (Ro1) in the heart, liver, and salivary gland of transgenic mice. Activation of Ro1 by spiradoline in the heart resulted in a $50 \%$ decrease in heart rate in less than $1 \mathrm{~min}$. As G-protein receptors are pivotal in numerous intracellular signaling pathways, this system could prove useful for dissecting their role in complex physiological processes (see also p. 131).

\section{A new twist on protein folding}

Obtaining efficient levels of folding has long been a problem in recombinant protein production. Because each protein has its particular requirements for efficient folding, purification protocols have to be designed on a case by case basis. Using a complex of GroEL chaperone, the DsbA foldase, and protein disulfide isomerase immobilized on agarose gel, Fersht and colleagues have provided a potential solution. They have refolded scorpion toxin $\mathrm{Cn} 5$, a protein traditionally very difficult to refold, even under optimal conditions (see pp. 136 and 187), to recover an $87 \%$ yield of $\mathrm{Cn} 5$ with $100 \%$ biological activity.

Research Briefs written by Andrew Marshall.

\title{
Bladder in a dish
}

For individuals with end-stage urinary bladder disease, often the only treatment is reconstructive surgery or bladder replacement. Because of the chronic shortage of suitable bladder donors, alternative sources of organs are needed. In this issue, Atala and colleagues describe an approach in which urothelial and smooth muscle cells were harvested from bladder biopsy specimens and seeded onto preformed bladder shaped polymers ( $\mathrm{pp}$. 133 and 149). The resultant urinary bladder substitute organs were then tranplanted into dogs whose native bladders had been excised. After only one month, the bladder substitute had developed normal morphology and function, with urinary function maintained for at least 11 months of the study.

\section{PDZ domains to order}

PDZ domains facilitate the assembly of multiprotein complexes that participate in intracellular signaling mechanisms. Thus far, only a limited number have been identified. In this issue, Schneider et al. create artificial PDZ domains that target orphan proteins using error-prone PCR and homologous

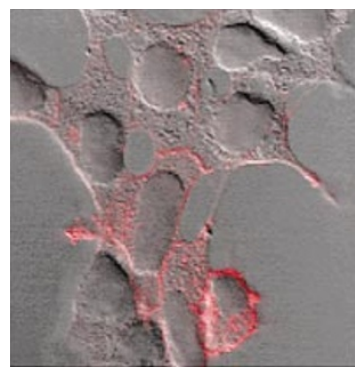
recombination in a yeast system (see pp. 132 and 170). Starting from a scaffold PDZ domain with no binding affinity to the target peptides, they isolated artificial PDZ domains that bound specifically and with high affinity to orphan targets. They went on to show that both target peptides and full-length proteins could be specifically targeted to the nucleus by attaching a nuclear localization sequence to the corresponding artificial PDZ domain.

\section{Low-lactose transgenic milk}

Hope may be on the horizon for the $70 \%$ of the adult population that finds cow's milk difficult to stomach. In these individuals, the presence of the sugar lactose leads to clinical symptoms, including abdominal pain, nausea, and diarrhea. To address this problem, dairy manufacturers offer milk in which lactose has been removed enzymatically postcollection. But these methods are often both expensive and time-consuming. An alternative approach is to express a lactase enzyme in the mammary gland that converts lactose to glucose and galactose (pp. 135 and 160). The resultant milk displays all the qualities and appearance of standard milk, but has a significantly reduced lactose content (as low as 15\% of control milk).

\section{Photopolymerizable bone substitute}

On p. 156, Langer and colleagues report a new family of photopolymerizable polyanhydrides with degradation times ranging from 1 week to nearly 1 year. When implanted into rats, molded, and cured using ultraviolet light, the polymer network adhered to the bone defect, with few histological signs of damage to the surrounding tissue. The polymers could have many applications, ranging from dentistry to orthopedics.

\section{Optimizing Mab function}

Improper or inadequate glycosylation of a monoclonal antibody produced in mammalian cell culture often results in poor effector function and disappointing clinical efficacy. Bailey and colleagues have addressed this problem by introducing into Chinese hamster ovary $(\mathrm{CHO})$ cells a tetracycline-regulated glycosyltransferase capable of incorporating appropriate oligosaccharides into an antineuroblastoma monoclonal antibody. When the antibody was expressed in these engineered $\mathrm{CHO}$ cells, antibody-dependent cellular cytotoxicity could be optimized according to the level of glycosyltransferase induction (p. 176).

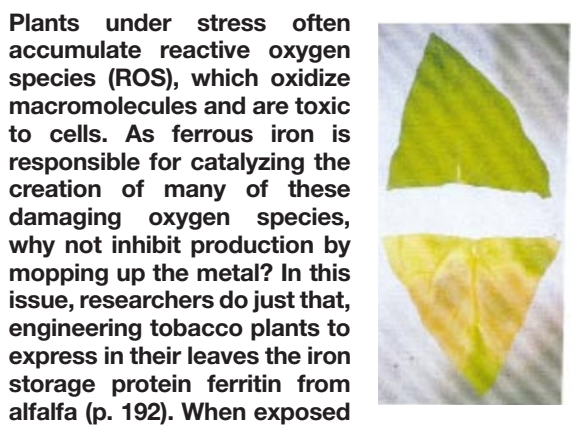
alfalfa (p. 192). When exposed to iron excess or paraquat herbicide treatment, these tobacco plants retained photosynthetic function and showed enhanced tolerance to necrotic damage by viral and fungal pathogens. 